\section{Lesiones cutáneas en mieloma múltiple. Descripción de un caso y revisión de la literatura}

\author{
CAMILA PEÑA O., XIMENA VALLADARES T., \\ ANA MARÍA GRAY H., MARÍA ELENA CABRERA C.
}

\section{Cutaneous involvement in multiple myeloma. Report of one case}

\begin{abstract}
Cutaneous involvement in multiple myeloma (MM) is extremely rare. It may correspond to plasmocytomas, secondary amyloidosis, paraneoplastic syndromes or drug reactions. We report a 75-year-old female with skin lesions, corresponding to extramedullary plasmacytomas (EPs), as a manifestation of MM progression. Most EPs appear in the upper airway and gastrointestinal tract, but have also been described in the genitourinary tract, lung, kidney, thyroid and in 2\% of cases, in the skin. Cutaneous lesions are generally associated with terminal stages of the disease and a poor prognosis.
\end{abstract}

(Rev Med Chile 2014; 142: 1603-1606)

Key words: Multiple Myeloma; Plasmacytoma; Skin, neoplasms.

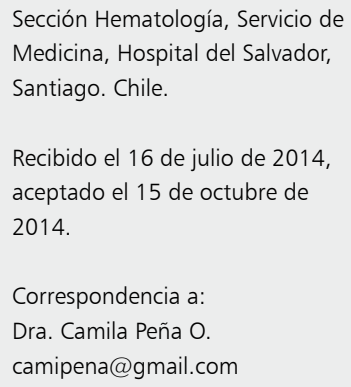

L a afectación cutánea en gammapatías monoclonales es extremadamente infrecuente. Puede observarse en cualquier neoplasia de células plasmáticas, tales como mieloma múltiple $(\mathrm{MM})$, leucemia de células plasmáticas, etc. Estas lesiones cutáneas pueden corresponder a diferentes entidades: plasmocitoma cutáneo (primario o secundario), amiloidosis asociada a mieloma múltiple, síndrome paraneoplásico o incluso pueden ser secundarias a drogas como el melfalán o el bortezomib. Dentro de los plasmocitomas, los más infrecuentes son los extramedulares secundarios a MM. Dado su baja frecuencia no se conoce con certeza la clínica, tratamiento adecuado ni pronóstico.

Se presenta un caso clínico de una paciente añosa con MM que presentó lesiones cutáneas como signo de progresión de su enfermedad.

\section{Caso clínico}

Presentamos el caso de una paciente de sexo femenino, de 75 años, con antecedentes de hi- pertensión e hipotiroidismo postquirúrgico (por bocio nodular). Consultó en agosto de 2008 por un cuadro de 9 meses de evolución caracterizado por fatigabilidad, síncopes a repetición y disnea progresiva. Dentro del estudio se pesquisó anemia severa normocítica normocrómica, con hemoglobina de $6,8 \mathrm{~g} / \mathrm{dL}$, leucocitos y plaquetas normales. Además destacó una VHS de $140 \mathrm{~mm} / \mathrm{h}$. Por sospecha de MM se continuó estudio destacando proteínas totales $14,6 \mathrm{~g} / \mathrm{dL}$, albúmina $2 \mathrm{~g} / \mathrm{dL}$, calcio corregido $10,8 \mathrm{mg} / \mathrm{dL}$, creatinina: $0,8 \mathrm{mg} / \mathrm{dL}, \mathrm{B} 2$ microglobulina $10,2 \mathrm{mg} / \mathrm{L}$. La electroforesis de proteínas reveló un peak monoclonal en gamma y se vio un aumento de inmunoglobulina IgG de $3.450 \mathrm{mg} / \mathrm{dl}$, siendo el resto de las Ig menores al rango normal. Se realizó un mielograma que mostró $25 \%$ de plasmoblastos y plasmocitos, algunos binucleados. La serie radiológica reveló imágenes líticas en calota y en columna lumbar. Por lo tanto, se hizo el diagnóstico de MM IgG, con índices pronósticos de Durie Salmon IIIA e ISS de 3 puntos.

Se inició tratamiento con hidratación, pamidronato y melfalán/prednisona, recuperándose la 
hipercalcemia. Recibió tratamiento con melfalán/ prednisona por 6 ciclos y radioterapia de columna lumbar, destacando en los controles ambulatorios mejoría en albúmina y calcemia, pero pancitopenia progresiva e infecciones urinarias a repetición. En marzo de 2009 estuvo hospitalizada por neumonía. En noviembre de 2009 evolucionó con mayor dolor óseo y postración. Además, llamó la atención la aparición de lesiones cutáneas nodulares, violáceas, dolorosas de 1,5 a $2 \mathrm{~cm}$ en cuero cabelludo, ángulo mandibular izquierdo, cuello y dorso (Figura 1). Se realizó una punción de las lesiones que mostró una celularidad constituida exclusivamente por células plasmáticas predominando proplasmocitos. La biopsia mostró igualmente infiltración por células plasmáticas. Debido a progresión de la enfermedad se cambió el tratamiento a talidomida/dexametasona, sin resultados. Se derivó a cuidados paliativos y la paciente falleció 2 meses después, por infección en mieloma refractario.

\section{Discusión}

Las lesiones cutáneas en MM ocurren sólo en raras ocasiones, por lo que su incidencia es desconocida. La literatura al respecto se basa sólo en revisión de casos clínicos. Las posibles causas de estas lesiones cutáneas se enumeran en la Tabla 1.
La amiloidosis secundaria a mieloma múltiple ocurre en $15 \%$, y se caracteriza por depósito extracelular de un material proteico fibrilar. Este sustituye el parénquima de órganos afectados y por ende su función. En 30\% la afectación es mucocutánea y frecuentemente es la primera manifestación. Se puede manifestar como púrpura periorbitario, pápulas o placas infiltrantes o infiltración esclerodermiforme. Más raramente ocurren lesiones bulosas ${ }^{1}$. El diagnóstico de certeza es mediante el estudio anatomopatológico. En nuestro caso descartó por aparecer en la biopsia una clara infiltración de células plasmáticas.

Los síndromes paraneoplásicos en MM también pueden manifestarse con lesiones cutáneas. Su incidencia varía entre 7 y $15 \%$ de los pacientes con cáncer ${ }^{2}$. En MM se han descrito casos de síndrome de Sweet o dermatosis neutrofílica febril

Tabla 1. Causas de lesiones cutáneas en mieloma múltiple

Plasmocitomas primarios

Plasmocitomas secundarios por contiguidad

Plasmocitomas secundarios metastásicos

Asociadas a Amiloidosis AL

Asociadas a síndromes paraneoplásicos

Secundarias a fármacos

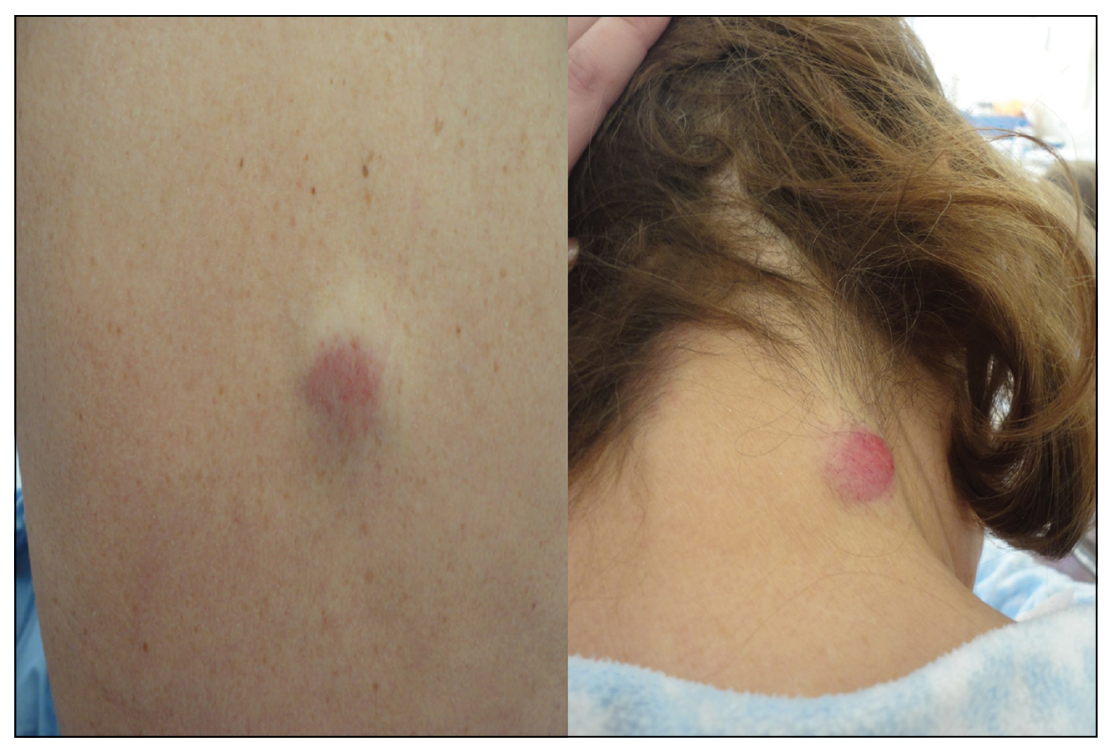

Figura 1. Imágenes de las lesiones cutáneas en la paciente. 
aguda $^{3}$, de escleredema ${ }^{4}$, de dermatosis bulosas ${ }^{5}$ y pioderma gangrenoso ${ }^{6}$, todos pocos frecuentes y de diferente presentación a la de nuestro caso.

Los fármacos como el melfalán pueden producir reacciones alérgicas cutáneas, aunque esto sucede raramente. Las lesiones tienen aspecto de erosión o rash, lo que no concuerda con nuestro caso. El bortezomib es actualmente el estándar de tratamiento y una de las drogas más usadas en MM. Si bien no se tuvo acceso a este fármaco en nuestro caso, es importante reconocerlo como posible causa de lesiones cutáneas. Entre sus efectos adversos se encuentran usualmente los gastrointestinales, como náuseas, vómitos; la neuropatía y la trombocitopenia, todos estos generalmente leves. Las lesiones cutáneas son más bien raras, aunque se han descrito pacientes con presentaciones clínicas muy variables, como rash purpúrico generalizado o placas eritematosas. El rash asociado a este fármaco es generalmente asintomático caracterizado por placas en el tronco, cuello y espalda. También se describe exantema morbiliforme, úlceras, nódulos eritematosos y pápulas. Ocurren generalmente durante el segundo ciclo y se resuelven en pocos días tras la suspensión del tratamiento ${ }^{7}$. En nuestro caso no se usó este fármaco.

Los plasmocitomas son otra causa de lesiones cutáneas en MM. Estos pueden ser primarios, es decir, sin asociación con otra neoplasia de células plasmáticas sistémica; o secundarios a ésta. Los plasmocitomas secundarios ocurren por contigüidad de una lesión ósea subyacente o, más raramente, plasmocitomas extramedulares $(\mathrm{PE})$ metastásicos. La mayoría de los PE se encuentran en la vía aérea alta y tracto gastrointestinal, aunque también se han descrito en tracto genitourinario, pulmón, riñón, tiroides y, raramente, en la piel. Los PE cutáneos son entidades inusuales, presentándose en alrededor de $2 \%{ }^{8}$.

Nuestro caso se trata de la más rara de las presentaciones de PE: plasmocitomas secundarios extramedulares metastásicos, ya que se observaron en una paciente con diagnóstico previo de MM y no correspondían a lesiones por contigüidad. Consiste generalmente en placas o nódulos subcutáneos de 1 a $5 \mathrm{~cm}$ en tórax o abdomen. También pueden encontrarse en rostro, cuello o extremidades$^{9}$. En nuestro caso se evidenciaron lesiones nodulares subcutáneas en cuero cabelludo, dorso, cuello y ángulo mandibular izquierdo. Ocurren generalmente en estadíos muy tardíos de la enfermedad de base, siendo raras las lesiones cutáneas como primera manifestación de la enfermedad. Algunos autores han postulado que la afectación cutánea específica en pacientes con MM no tiene lugar hasta que la masa tumoral total alcanza un peso de alto riesgo, que se considera de $2 \mathrm{a}^{2} \mathrm{~kg}^{10}$. Indican un comportamiento agresivo y corta sobrevida $^{11,12}$. De hecho, luego del diagnóstico, la expectativa de vida es menor de 12 meses $^{13}$, independiente de la quimioterapia administrada ${ }^{14}$. Nuestro caso confirma el mal pronóstico de la aparición de estas lesiones cutáneas secundarias, ya que la paciente falleció sólo 2 meses luego de su diagnóstico.

La fisiopatología no está del todo clara, pero podría deberse a una disminución de expresión de moléculas de adhesión, como VLA-4 y CD44, sumado a una pérdida de CD56, lo que facilitaría la diseminación hematógena, al disminuir la adherencia de las células plasmáticas a la médula ósea ${ }^{15,16}$. En cuanto a la histopatología, los plasmocitomas cutáneos se dividen en dos grupos: los con patrón nodular y con patrón intersticial. El patrón nodular muestra en la dermis grupos de células neoplásicas cohesivas. En el patrón intersticial, las células forman cordones delgados entre las bandas de colágeno. La inmunohistoquímica ayuda a diferenciar un proceso neoplásico de uno reactivo. Muestra generalmente células con marcadores CD138 positivas, con CD38 y CD79 a variable. El CD19 y CD20 son negativos. El CD56 puede estar aberrantemente positivo ${ }^{17}$. En nuestro caso no se realizó inmunohistoquímica por interpretarse la histología como diagnóstica.

El estudio más grande de lesiones cutáneas en MM de un único centro da cuenta de 40 casos $^{18}$. En este se vio que desde el punto de vista clínico, la morfología más frecuente de las lesiones son múltiples pápulas o nódulos de consistencia firme, en distintas áreas corporales, preferentemente localizados en la pared anterior del tórax, pared anterior del abdomen y dorso. En cuanto a la inmunoglobulina (Ig) comprometida, 19 pacientes tenían MM IgG, 18 MM IgA y 2 pacientes MM IgD. En la histopatología, el patrón nodular fue el más frecuente, en 24 casos, mientras que el patrón intersticial sólo estaba presente en 13.

En este mismo estudio además se recopilaron datos de 92 pacientes, correspondientes a todos los casos descritos en la literatura médica hasta 
el momento de su publicación ${ }^{18}$. En este, $63 \%$ de los casos fue IgG, 25\% fue IgA, en 6 casos (10\%) fue IgD y en uno (1\%) se trataba de un mieloma IgM. No hubo relación entre la Ig y pronóstico.

El manejo es controversial. En general consiste en quimioterapia más cirugía o radioterapia, todas con pobres resultados. La radioterapia puede usarse en forma paliativa ${ }^{19}$. En este caso se intentó utilizar quimioterapia, con dexametasona y talidomida, sin resultados. Se debe destacar que la terapia standard es el bortezomib, al que no tuvimos acceso. Si bien los plasmocitomas cutáneos metastásicos implican mal pronóstico, esto podría haber influido en la nula respuesta clínica obtenida. Debido a la rápida progresión y fallecimiento de la paciente no fue posible el uso de la radioterapia.

En conclusión, presentamos el caso de una paciente con MM y lesiones cutáneas como manifestación terminal de la enfermedad. Nos pareció interesante documentar esta rara manifestación y corroborar su mal pronóstico.

\section{Referencias}

1. Vega Gutiérrez J, Martínez Fernández M, Sánchez Sambucety P, Santos Sales Valiente J, Rodríguez Prieto MA, Cutaneous Manifestations of Myeloma-Associated Systemic Amyloidosis, Med Cutan Iber Lat Am 2004; 32 (5): 211-4.

2. Frías G, Hierro S, Manifestaciones cutáneas en pacientes con alteraciones hematológicas. Dermatología Rev Mex 2007; 51 (4): 142-8.

3. Tazi I, Nafil H, Mahmal L. Immunoglobulin a multiple myeloma associated with sweet syndrome, J Cancer Res Ther 2012; 8 (4): 652-3.

4. Santos-Juanes J, Osuna CG, Iglesias JR, De Quiros JF, del Río JS. Treatment with chemotherapy of scleredema associated with Ig A myeloma. Int J Dermatol 2001; 40 (11): 720-1.

5. Battistella M, Hickman G, Petit A, Asli B, Bouaziz JD, Janin A, et al. Acquired bullous dermatosis associated with IgA multiple myeloma. Indian J Dermatol Venereol Leprol 2013; 79 (5): 719-20.

6. Yoon YH, Cho WI, Seo SJ. Case of multiple myeloma associated with extramedullary cutaneous plasmacytoma and pyoderma gangrenosum. Int J Dermatol 2006; 45:
594-7.

7. Wu KL, Heule F, Lam K, Sonneveld P. Pleomorphic presentation of cutaneous lesions associated with the proteasome inhibitor bortezomib in patients with multiple myeloma, J Am Acad Dermatol 2006; 55 (5): 897-900.

8. Bayer-Garner IB, Smoller BR. The spectrum of cutaneous disease in multiple myeloma. J Am Acad Dermatol 2003; 48: 497-507.

9. Álvarez-Twose I, Vano-Galvan S, Calvo-Villas J, Carreter E, Piqué E, Palacios S. Metastatic cutaneous plasmacytoma presenting as a perianal giant mass, Derm online Journal 2008; 14 (9): 17.

10. Stankler L, Davidson JF. Multiple extramedullary plasmacytomas of the skin: case report with a note on prognosis. Br J Dermatol 1974; 90: 217-21.

11. Almeida Pereira M, Baudrier T, Costa A, Magalhães J, Azevedo F. Cutaneous metastatic plasmacytomas with tropism for a previously injured limb, Derm Online Journal 2008; 14 (9): 16.

12. Requena L, Kutzner H, Palmedo G, Calonje E, Requena C, Pérez G, et al, Cutaneous involvement in multiple Myeloma, Arch Dermatol 2003; 139 (4): 475-86.

13. Ballester-Martínez MA, González-García C, Fleta-Asín B, Burdaspal-Moratilla A, Santón-Roldán A, JiménezGómez N, et al. Cutaneous nodules as a diagnostic clue in multiple mieloma, Am J Dermatopathol 2013; 35 (3): 377-80.

14. Sellami RD, Sassi S, Mrad K, Abess I, Driss M, Ben Romdhane K. Plasmocytome cutané primitif Ann Pathol 2007; 27 (2): 130-2.

15. Vande Broek I, Vanderkerken K, Van Camp B, et al. Extravasation and homing mechanisms in multiple myeloma. Clin Exp Metastasis 2008, 25: 325-34.

16. Bladé J, Fernández de Larrea C, Rosiñol L, Cibeira MT, Jiménez R, Powles R. Soft-Tissue Plasmacytomas in Multiple Myeloma: Incidence, Mechanisms of Extramedullary Spread, and Treatment Approach, J Clin Oncol 2011; 29 (28): 3805-12.

17. Bayer-Garner IB, Joseph L, Sanderson RD, Smoller BR. Expression of syndecan-1 is a sensitive marker for cutaneous plasmacytoma. J Cutan Pathol 2003; 30: 18-22.

18. Requena L. Afectación cutánea específica en pacientes con mieloma múltiple. Estudio clínico-patológico, inmunohistoquímico y citogenético de 40 casos; Actas Dermosifiliogr 2005; 96 (7): 424-40.

19. Nguyen SK, Dagnault A. Radiotherapy for multiple myeloma with skin involvement. Curr Oncol 2010; 17 (5): 74-7. 